\title{
$\left.{ }_{\text {SCIENCE }}^{\text {MATRIX }}\right\}$ Mascot Search Results
}

\section{Protein View}

Match to: FLID_SALTY Score: 135 Expect: 4e-010

Flagellar hook-associated protein 2 OS=Salmonella typhimurium (strain LT2 / SGSC1412 / ATCC 700720 ) GN=fliD PE=1 SV=2

Nominal mass $\left(M_{r}\right)$ : 49805; Calculated pI value: $\mathbf{5 . 2 7}$

NCBI BLAST search of FLID SALTY against $n r$

Unformatted sequence string for pasting into other applications

Taxonomy: Salmonella enterica subsp. enterica serovar Typhimurium str. LT2

Variable modifications: Carbamidomethyl (C), Oxidation (M)

Cleavage by Trypsin: cuts C-term side of KR unless next residue is $P$

Number of mass values searched: 87

Number of mass values matched: $\mathbf{2 4}$

Sequence Coverage: 61\%

Matched peptides shown in Bold Red

1 MASISSLGVG SNLPLDQLLT DLTKNEKGRL TPITKQQSAN SAKLTAYGTL

51 KSALEKFQTA NTALNKADLF KSTVASSTTE DLKVSTTAGA AAGTYKINVT

101 QLAAAOSLAT KTTFATTKEO LGDTSVTSRT IKIEOPGRKE PLEIKLDKGD 151 TSMEAIRDAI NDADSGIAAS IVKVKENEFO LVLTANSGTD NTMKITVEGD

201 TKLNDLLAYD STTNTGNMQE LVKAENAKLN VNGIDIERQS NTVTDAPQGI 251 TLTLTKKVTD ATVTVTKDDT KAKEAIKSWV DAYNSLVDTF SSLTKYTAVE

301 PGEEASDKNG ALLGDSVVRT IOTGIRAOFA NSGSNSAFKT MAEIGITODC

351 TSGKLKIDDD KLTKVLKDNT AAARELLVGD GKETGITTKI ATEVKSYLAD

401 DGIIDNAQDN VNATLKSLTK QYLSVSNSID ETVARYKAQF TQLDTMMSKL

451 NNTSSYLTQQ FTAMNKS

Show predicted peptides also

Sort Peptides By $\odot$ Residue Number $\bigcirc$ Increasing Mass $\bigcirc$ Decreasing Mass

$\begin{array}{rrrr}\text { Start - End } & \text { Observed } & \text { Mr (expt) } & \text { Mr (calc) } \\ 2-24 & 2342.2781 & 2341.2708 & 2341.2740 \\ 57-66 & 1107.5633 & 1106.5560 & 1106.5720 \\ 97-111 & 1528.8687 & 1527.8614 & 1527.8620 \\ 119-129 & 1192.5864 & 1191.5791 & 1191.5732 \\ 133-138 & 699.3677 & 698.3604 & 698.3711 \\ 149-173 & 2520.2265 & 2519.2192 & 2519.2173 \\ 149-173 & 2536.2191 & 2535.2118 & 2535.2122 \\ 203-223 & 2340.1412 & 2339.1340 & 2339.1315 \\ 203-223 & 2356.1374 & 2355.1302 & 2355.1264 \\ 229-238 & 1142.6162 & 1141.6089 & 1141.6091 \\ 239-256 & 1887.9987 & 1886.9915 & 1886.9949 \\ 258-271 & 1493.7317 & 1492.7245 & 1492.7621 \\ 278-295 & 2032.9838 & 2031.9765 & 2031.9789 \\ 309-319 & 1100.6040 & 1099.5968 & 1099.5986 \\ 320-326 & 788.4555 & 787.4482 & 787.4552 \\ 327-339 & 1328.6252 & 1327.6179 & 1327.6157 \\ 340-356 & 1765.7321 & 1764.7249 & 1764.8928 \\ 396-416 & 2250.0839 & 2249.0766 & 2249.0811 \\ 421-435 & 1681.8376 & 1680.8304 & 1680.8319 \\ 436-449 & 1707.7717 & 1706.7644 & 1706.8008 \\ 438-449 & 1400.6553 & 1399.6480 & 1399.6476 \\ 438-449 & 1416.6540 & 1415.6468 & 1415.6425 \\ 450-466 & 1960.9385 & 1959.9312 & 1959.9360 \\ 450-466 & 1976.9327 & 1975.9255 & 1975.9309\end{array}$

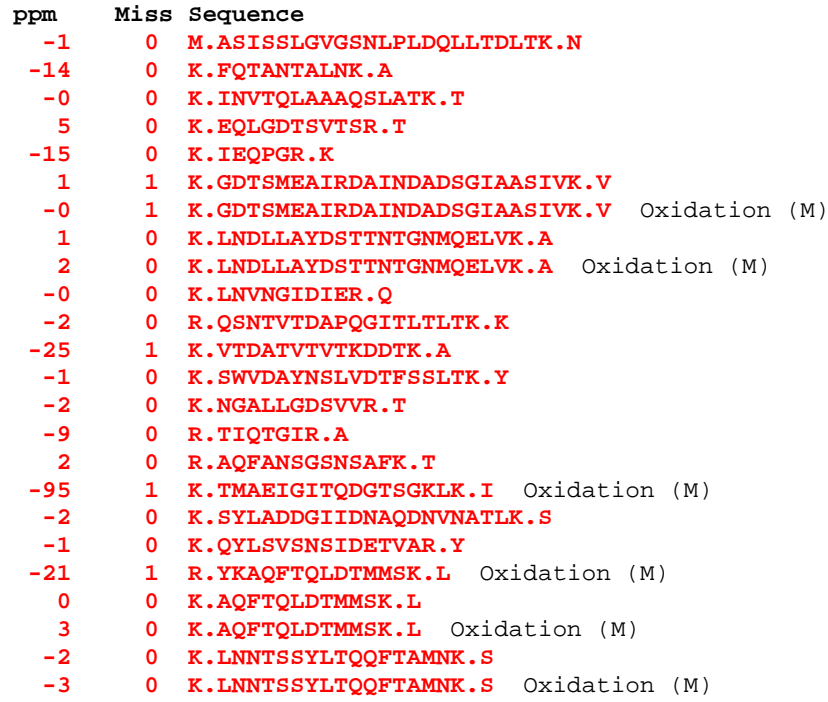

No match to: $634.2548,644.0073,662.2896,832.3028,855.0469,881.2513,1036.5412,1060.0607,1109.5032,1143.6116,1193.6$
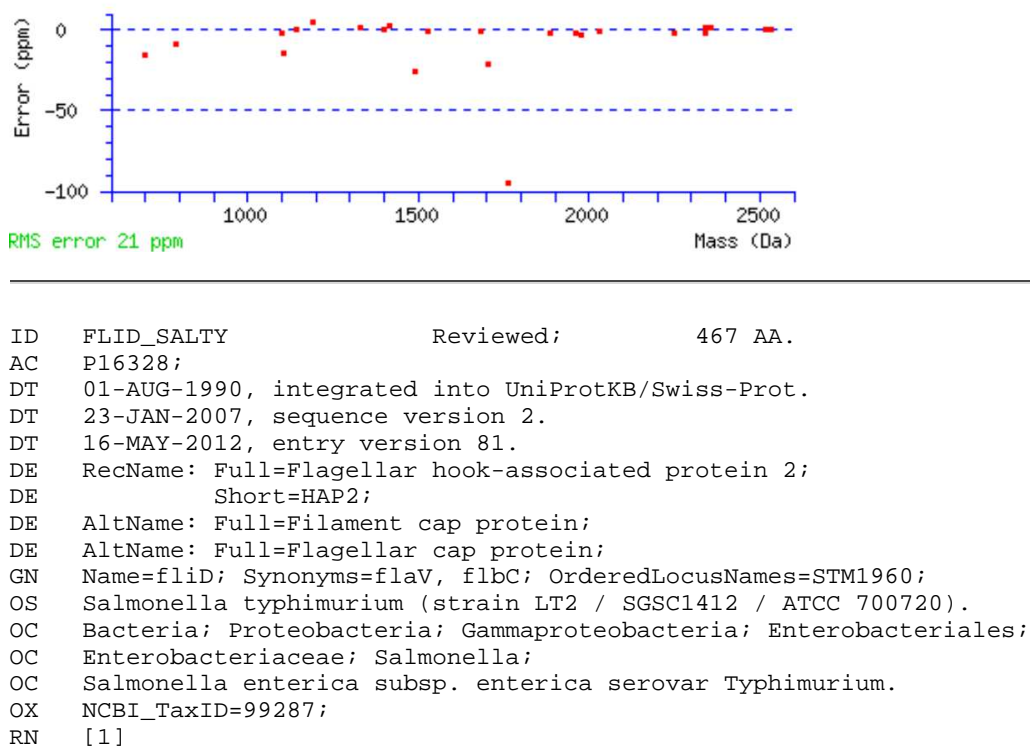


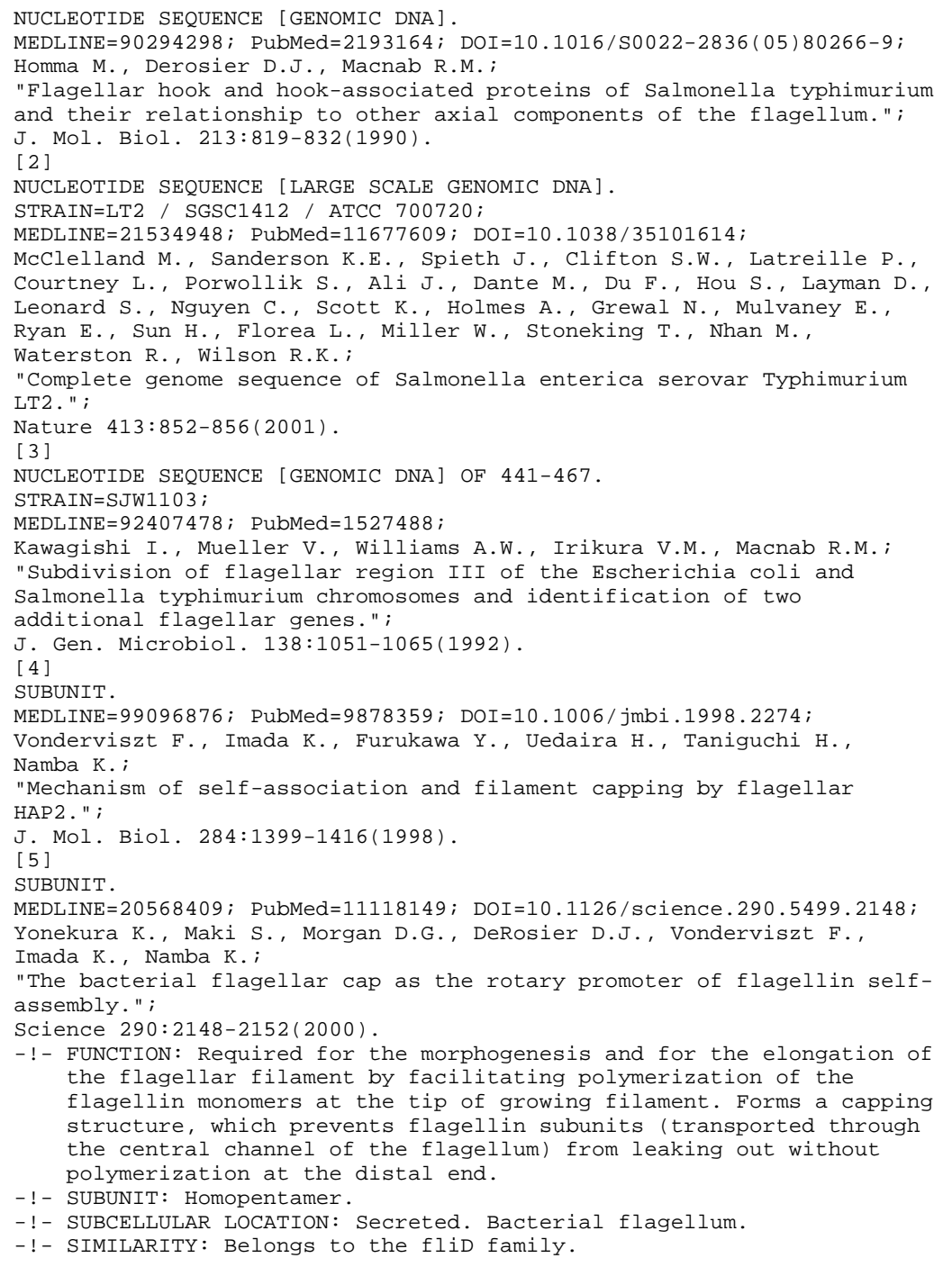

Copyrighted by the UniProt Consortium, see http://www.uniprot.org/terms Distributed under the Creative Commons Attribution-NoDerivs License

EMBL; X51740; CAA36030.1; -; Genomic_DNA.

EMBL; AE006468; AAL20872.1; -; GenomiC_DNA.

EMBL; M85241; AAA27076.1; -; Genomic_DNA.

PIR; S10364; S10364.

RefSeq; NP_460913.1; NC_003197.1.

ProteinModelPortal; P16328; -

PRIDE; P16328; - .

GeneID; 1253481 ; -

GenomeReviews; AE006468_GR; STM1960.

KEGG; stm:STM1960; - .

PATRIC; 32382481; VBISalEnt20916_2076.

eggNOG; COG1345; -

HOGENOM; HOGO00254632; -

$\mathrm{KO}$; K02407; -.

OMA; FRTQISA; -

ProtClustDB; PRK08032; -

BioCyc; STYP99287:STM1960-MONOMER; - .

GO; GO:0009288; C:bacterial-type flagellum; IEA:UniProtKB-SubCell.

GO; GO:0005576; C:extracellular region; IEA:UniProtKB-SubCell.

GO; GO:0007155; P:cell adhesion; IEA: InterPro.

GO; GO:0006928; P:cellular component movement; IEA:InterPro.

GO; GO:0009296; P:flagellum assembly; IEA:InterPro.

InterPro; IPR010810; Flagellin_hook_IN_motif.

InterPro; IPR010809; FliD_C.

InterPro; IPR003481; FliD_N.

Pfam; PF07196; Flagellin_IN; 1 .

Pfam; PF07195; FliD_C; 1 .

Pfam; PF02465; FliD_N; 1 .

1: Evidence at protein level;

Bacterial flagellum; Coiled coil; Complete proteome

Reference proteome; Secreted.

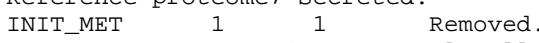

CHAIN $\quad 2 \quad 467 \quad$ Flagellar hook-associated protein 2.

/FTId=PRO_0000177024.

COILED $411 \quad 439 \quad$ Potential.

MASISSLGVG SNLPLDQLLT DLTKNEKGRL TPITKOOSAN SAKLTAYGTL KSALEKFQTA

NTALNKADLF KSTVASSTTE DLKVSTTAGA AAGTYKINVT QLAAAQSLAT KTTFATTKEQ LGDTSVTSRT IKIEQPGRKE PLEIKLDKGD TSMEAIRDAI NDADSGIAAS IVKVKENEFQ LVLTANSGTD NTMKITVEGD TKLNDLLAYD STTNTGNMQE LVKAENAKLN VNGIDIERQS NTVTDAPQGI TLTLTKKVTD ATVTVTKDDT KAKEAIKSWV DAYNSLVDTF SSLTKYTAVE PGEEASDKNG ALLGDSVVRT IOTGIRAOFA NSGSNSAFKT MAEIGITODG TSGKLKIDDD KLTKVLKDNT AAARELLVGD GKETGITTKI ATEVKSYLAD DGIIDNAQDN VNATLKSLTK 
